\title{
Oriented hypergraphic matrix-tree type theorems and bidirected minors via Boolean order ideals
}

\author{
Lucas J. Rusnak ${ }^{1}$. Ellen Robinson ${ }^{1}$. Martin Schmidt ${ }^{1}$. Piyush Shroff ${ }^{1}$ \\ Received: 8 November 2017 / Accepted: 11 June 2018 / Published online: 26 June 2018 \\ (c) Springer Science+Business Media, LLC, part of Springer Nature 2018
}

\begin{abstract}
Restrictions of incidence preserving path maps produce oriented hypergraphic All Minors Matrix-tree Theorems for Laplacian and adjacency matrices. The images of these maps produce a locally signed graphic, incidence generalization, of cycle covers and basic figures that correspond to incidence- $k$-forests. When restricted to bidirected graphs, the natural partial ordering of maps results in disjoint signed Boolean lattices whose minor calculations correspond to principal order ideals. As an application, (1) the determinant formula of a signed graphic Laplacian is reclaimed and shown to be determined by the maximal positive-circle-free elements, and (2) spanning trees are equivalent to single-element order ideals.
\end{abstract}

Keywords Matrix-tree theorem - Laplacian matrix - Signed graph · Bidirected graph · Oriented hypergraph

Mathematics Subject Classification 05C50 - 05C65 - 05C22

\section{Introduction}

An oriented hypergraph is a signed incidence structure that first appeared in [21] to study applications to VLSI via minimization and logic synthesis, and generalize Kirchhoff's laws [22,24]. This incidence-based approach allows for Laplacian, spectral, and balanced, graph-theoretic theorems to be extended to hypergraphs using their locally signed graphic structure $[7,17,18]$. Moreover, the concepts of a balanced hypergraph $[2,20]$ and a balanced $\{0, \pm 1\}$-matrix $[9,10,13]$ can simultaneously be studied through their oriented hypergraphic structure [19].

Sachs' Theorem [11] characterizes the coefficients of the characteristic polynomial of the adjacency matrix by generalizing the concept of a cycle cover of a graph and has

\footnotetext{
$凶$ Lucas J. Rusnak

Lucas.Rusnak@txstate.edu

1 Texas State University, San Marcos, USA
} 
long known application in the study of molecular orbitals [14]. Sachs' Theorem was recently generalized to signed graphs in [1], and to oriented hypergraphs in [6]. We show that the incidence generalization of cycle covers (called contributors) obtained in [6] allows for the hypergraphic generalization of the All Minors Matrix-tree Theorem of Chaiken in $[4,5]$. Moreover, when restricted to bidirected graphs, the contributors align with Chaiken's $k$-forests.

Section 2 collects the necessary oriented hypergraphic background to present the All Minors Matrix-tree Theorem as a consequence of the oriented hypergraphic Sachs' Theorem in [6] - the coefficients of the characteristic polynomial are the diagonal minors, are determined by contributor sums, and a similar proof can be used to obtain any minor.

Contributor maps are specialized to Laplacians of bidirected graphs in Sect. 3. A natural partial ordering of contributors is introduced where each associated equivalence class (called activation classes) is Boolean; this is done by introducing incidence packing and unpacking operations. If an oriented hypergraph contains edges of size larger than 3, then unpacking is not well defined, and the resulting equivalence classes need not be lattices. Activation classes are further refined via iterated principal order ideals in order to examine minors of the Laplacian.

Section 4 examines the contributors in the adjacency completion of a bidirected graph to obtain a restatement of the All Minors Matrix-tree Theorem in terms of sub-contributors (as opposed to restricted contributors). This implies there is a universal collection of contributors (up to resigning) which determines the minors of all bidirected graphs that have the same injective envelope-see [15] for more on the injective envelope. These sub-contributors determine permanents/determinants of the minors of the original bidirected graph and are activation equivalent to the forest-like objects in [4]. Additionally, the standard determinant of the signed graphic Laplacian is presented as a sum of maximal contributors, while the first minors of the Laplacian contain a subset of contributors that are activation equivalent to spanning trees.

While the techniques introduced for bidirected graphs do not readily extend to all oriented hypergraphs, they bear a remarkable similarity to Tutte's development of transpedances in [23] — indicating the possibility of a locally signed graphic interpretation of transpedance theory. Since contributor sets produce the finest possible set of objects signed $\{0, \pm 1\}$ whose sum produces the permanent/determinant it is natural to ask what classes of graphs achieve specific permanent/determinant values. Additionally, recent work on vertex-weighted Laplacians [8], graph dynamics [3], and oriented spanning trees and sandpile groups [16], seem to have natural oriented hypergraphic analogs.

\section{Preliminaries and the matrix-tree theorem}

\subsection{Oriented hypergraph basics}

A condensed collection of definitions are provided in this subsection to improve readability, for a detailed introduction to the definitions the reader is referred to [6]. An oriented hypergraph is a quintuple $(V, E, I, \iota, \sigma)$ where $V, E$, and $I$ are disjoint sets 
of vertices, edges, and incidences, with incidence function $\iota: I \rightarrow V \times E$, and incidence orientation function $\sigma: I \rightarrow\{+1,-1\}$. A bidirected graph is an oriented hypergraph with the property that for each $e \in E,\left|\left\{i \in I \mid\left(\operatorname{proj}_{E} \circ \iota\right)(i)=e\right\}\right|=2$, and can be regarded as an orientation of a signed graph (see $[25,26])$ where the sign of an edge $e$ is

$$
\operatorname{sgn}(e)=-\sigma(i) \sigma(j)
$$

where $i$ and $j$ are the incidences containing $e$.

A backstep of $G$ is an embedding of $\vec{P}_{1}$ into $G$ that is neither incidence-monic nor vertex-monic; a loop of $G$ is an embedding of $\vec{P}_{1}$ into $G$ that is incidence-monic but not vertex-monic; a directed adjacency of $G$ is an embedding of $\vec{P}_{1}$ into $G$ that is incidence-monic. A directed weak walk of length $k$ in $G$ is the image of an incidence preserving embedding of a directed path of length $k$ into $G$. Conventionally, a backstep is a sequence of the form $(v, i, e, i, v)$, a loop is a sequence of the form $(v, i, e, j, v)$, and an adjacency is a sequence of the form $(v, i, e, j, w)$ where $\iota(i)=(v, e)$ and $\iota(j)=(w, e)$. The opposite embedding is image of the reversal of the initial directed path, while the non-directed version is the set on the sequence's image.

The sign of a weak walk is defined as

$$
\operatorname{sgn}(W)=(-1)^{k} \prod_{h=1}^{2 k} \sigma\left(i_{h}\right),
$$

which implies that for a path in $G$ the product of the adjacency signs of the path is equal to the sign of the path calculated as a weak walk.

\subsection{The matrix-tree theorem}

It was shown in [18] that the $(v, w)$-entry of the oriented incidence Laplacian are the negative weak walks of length 1 from $v$ to $w$ minus the number of positive weak walks of length 1 from $v$ to $w$. This was restated in [6] as follows:

Theorem 2.1 The $(v, w)$-entry of $\mathbf{L}_{G}$ is $\sum_{\omega \in \Omega_{1}}-\operatorname{sgn}\left(\omega\left(\vec{P}_{1}\right)\right)$, where $\Omega_{1}$ is the set of all incidence preserving maps $\omega: \vec{P}_{1} \rightarrow G$ with $\omega(t)=v$ and $\omega(h)=w$.

A contributor of $G$ is an incidence preserving function $c: \coprod_{v \in V} \vec{P}_{1} \rightarrow G$ with $p\left(t_{v}\right)=v$ and $\left\{p\left(h_{v}\right) \mid v \in V\right\}=V$. A strong-contributor of $G$ is an incidence-monic contributor of $G$-i.e., the backstep-free contributors of $G$. Let $\mathfrak{C}(G)(\operatorname{resp} . \mathfrak{S}(G))$ denote the sets of contributors (resp. strong contributors) of $G$. In [6] contributors provided a finest count to determine the permanent/determinant of Laplacian and adjacency matrices and their characteristic polynomials of any integral matrix as the incidence matrix of the associated oriented hypergraph. The values ec $(c), o c(c), p c(c)$, and $n c(c)$ denote the number of even, odd, positive, and negative circles in the image of contributor $c$. Additionally, the sets $\mathfrak{C}_{\geq 0}(G)$ (resp. $\mathfrak{C}_{=0}(G)$ ) denote the set of all contributors with at least 0 (resp. exactly 0 ) backsteps. 
Theorem 2.2 ([6]) Let $G$ be an oriented hypergraph with adjacency matrix $\mathbf{A}_{G}$ and Laplacian matrix $\mathbf{L}_{G}$, then

1. $\operatorname{perm}\left(\mathbf{L}_{G}\right)=\sum_{c \in \mathfrak{C}_{\geq 0}(G)}(-1)^{o c(c)+n c(c)}$,

2. $\operatorname{det}\left(\mathbf{L}_{G}\right)=\sum_{c \in \mathfrak{C}_{\geq 0}(G)}(-1)^{p c(c)}$,

3. $\operatorname{perm}\left(\mathbf{A}_{G}\right)=\sum_{c \in \mathfrak{C}_{=0}(G)}(-1)^{n c(c)}$,

4. $\operatorname{det}\left(\mathbf{A}_{G}\right)=\sum_{c \in \mathfrak{C}_{=0}^{*}(G)}(-1)^{e c(c)+n c(c)}$.

For a $V \times V$ matrix $\mathbf{M}$, let $U, W \subseteq V$, define $[\mathbf{M}]_{(U ; W)}$ be the minor obtained by striking out rows $U$ and columns $W$ from $\mathbf{M}$. Let $\mathfrak{C}(U ; W ; G)$ be the set of all subcontributors of $G$ with $c: \bigsqcup_{u \in \bar{U}} \vec{P}_{1} \rightarrow G$ with $p\left(t_{u}\right)=u$ and $\left\{p\left(h_{u}\right) \mid u \in \bar{U}\right\}=$ $\bar{W}$. Define $\mathfrak{S}(U ; W ; G)$ analogously for strong contributors. Let the values en $(c)$, on $(c), p n(c)$, and $n n(c)$ denote the number of even, odd, positive, and negative, nonadjacency-trivial components (paths or circles) in the image of $c$.

Theorem 2.3 Let $G$ be an oriented hypergraph with adjacency matrix $\mathbf{A}_{G}$ and Laplacian matrix $\mathbf{L}_{G}$, then

1. $\operatorname{perm}\left(\left[\mathbf{L}_{G}\right]_{(U ; W)}\right)=\sum_{c \in \mathfrak{C}(U ; W ; G)}(-1)^{o n(c)+n n(c)}$,

2. $\operatorname{det}\left(\left[\mathbf{L}_{G}\right]_{(U ; W)}\right)=\sum_{c \in \mathfrak{C}(U ; W ; G)} \varepsilon(c) \cdot(-1)^{o n(c)+n n(c)}$,

3. $\operatorname{perm}\left(\left[\mathbf{A}_{G}\right]_{(U ; W)}\right)=\sum_{c \in \mathfrak{S}(U ; W ; G)}(-1)^{n n(c)}$,

4. $\operatorname{det}\left(\left[\mathbf{A}_{G}\right]_{(U ; W)}\right)=\sum_{c \in \mathfrak{S}(U ; W ; G)} \varepsilon(c) \cdot(-1)^{e n(c)+n n(c)}$.

where $\varepsilon(c)$ is the number of inversions in the natural bijection from $\bar{U}$ to $\bar{W}$.

The proof of Theorem 2.3 is analogous to Theorem 4.1.1 in [6] using the bijective definitions of permanent/determinant.

The value of $\varepsilon(c)$ can be modified to count circle and paths separately, as the circle components simplify identical to the works in [6], thus part (2) of Theorem 2.3 can be restated as follows:

Theorem 2.4 Let $G$ be an oriented hypergraph with adjacency matrix $\mathbf{A}_{G}$ and Laplacian matrix $\mathbf{L}_{G}$, then

$$
\operatorname{det}\left(\left[\mathbf{L}_{G}\right]_{(U ; W)}\right)=\sum_{c \in \mathfrak{C}(U ; W ; G)} \varepsilon^{\prime}(c) \cdot(-1)^{p c(c)} \cdot(-1)^{o p(c)+n p(c)}
$$

where $\varepsilon^{\prime}(c)$ is the number of inversions in paths parts of the natural bijection from $\bar{U}$ to $\bar{W}$.

Comparing, the nonzero elements of $\mathfrak{C}(U ; W ; G)$ are the Chaiken-type structures in [4] with multiplicities replaced with backstep maps. 


\section{Contributor structure of bidirected graphs}

\subsection{Pre-contributors and incidence packing}

An oriented hypergraph in which every edge has exactly 2 incidences is a bidirected graph and can be regarded as orientations of signed graphs (see [12,25,26]).

Throughout this section, $G$ is a bidirected graph in which every connected component contains at least one adjacency, and $\vec{P}_{1}$ is a directed path of length 1 . A pre-contributor of $G$ is an incidence preserving function $p: \bigsqcup_{v \in V} \vec{P}_{1} \rightarrow G$ with $p\left(t_{v}\right)=v$. That is, the disjoint union of $|V|$ copies of $\vec{P}_{1}$ into $G$ such that every tail-vertex labeled by $v$ is mapped to $v$.

Consider a pre-contributor $p$ with $p\left(t_{v}\right) \neq p\left(h_{v}\right)$ for vertex $v \in V$. Packing $a$ directed adjacency of a pre-contributor p into a backstep at vertex $v$ is a pre-contributor $p_{v}$ such that $p_{v}=p$ for all $u \in V \backslash v$, and for vertex $v$

$$
\begin{aligned}
p\left(\left(\vec{P}_{1}\right)_{v}\right) & =(v, i, e, j, w), i \neq j, \\
\text { and } p_{v}\left(\left(\vec{P}_{1}\right)_{v}\right) & =(v, i, e, i, v) .
\end{aligned}
$$

Thus, the head-incidence and head-vertex of adjacency $p\left(\left(\vec{P}_{1}\right)_{v}\right)$ are identified to the tail-incidence and tail-vertex.

Unpacking a backstep of a pre-contributor p into an adjacency out of vertex $v$ is a pre-contributor $p^{v}$ is defined analogously but for vertex $v$, the head-incidence and head-vertex of backstep $p\left(\left(\vec{P}_{1}\right)_{v}\right)$ are identified to the unique incidence and vertex that would complete the adjacency in bidirected graph $G$. Note that this is unique for a bidirected graph since every edge has exactly two incidences, but this is not the case in if there are edges of size greater than 2 .

For a bidirected graph $G$ and vertex $v$, let $\mathfrak{P}(G)$ be the set of all pre-contributors of $G, \mathfrak{P}_{v}(G)$ be the set of pre-contributors with a backstep at $v$, and let $\mathfrak{P}^{v}(G)$ be the set of pre-contributors with a directed adjacency from $v$.

Lemma 3.1 Packing and unpacking are inverses between $\mathfrak{P}_{v}$ and $\mathfrak{P}^{v}$.

Proof By definition $\left(p_{v}\right)^{v}=p$ and $\left(p^{v}\right)_{v}=p$ for appropriate contributors in $\mathfrak{P}^{v}$ or $\mathfrak{P}_{v}$.

Lemma 3.2 Packing is commutative.

Proof Let $p \in \mathfrak{P}^{v} \cap \mathfrak{P}^{w}, p_{v w}:=p_{w} \circ p_{v}$, and $p_{w v}:=p_{v} \circ p_{w}$. By definition, $p_{v w}=p_{w v}$ for all $\left(\vec{P}_{1}\right)_{u}$ with $u \in V \backslash\{v, w\}$. For vertices $v$ and $w$,

$$
\begin{aligned}
p_{v}\left(\left(\vec{P}_{1}\right)_{w}\right) & =p\left(\left(\vec{P}_{1}\right)_{w}\right), \\
\text { and } p_{w}\left(\left(\vec{P}_{1}\right)_{v}\right) & =p\left(\left(\vec{P}_{1}\right)_{v}\right)
\end{aligned}
$$


Giving,

$$
\begin{aligned}
p_{v w}\left(\left(\vec{P}_{1}\right)_{w}\right) & =p_{w}\left(\left(\vec{P}_{1}\right)_{w}\right)=p_{w v}\left(\left(\vec{P}_{1}\right)_{w}\right) \\
\text { and } p_{v w}\left(\left(\vec{P}_{1}\right)_{v}\right) & =p_{v}\left(\left(\vec{P}_{1}\right)_{v}\right)=p_{w v}\left(\left(\vec{P}_{1}\right)_{v}\right)
\end{aligned}
$$

Lemma 3.3 Unpacking is commutative.

Proof Proof is identical to packing after reversing subscript and superscripts.

\subsection{Contributors and activation}

A contributor of $G$ is a pre-contributor where $\left\{p\left(h_{v}\right) \mid v \in V\right\}=V$.

For each $c \in \mathfrak{C}(G)$, let $t c(c)$ be the total number of circles in $c$; a degenerate 2-circle (a closed 2-weak walk) is considered a circle, while a degenerate 1-circle (a backstep) is not. Activating a circle of contributor $c$ is a minimal sequence of unpackings that results in a new contributor $c^{\prime}$ such that $t c(c)=t c\left(c^{\prime}\right)-1$. Deactivating a circle of contributor $c$ is a minimal sequence of packings that results in a new contributor $c^{\prime \prime}$ such that $t c(c)=t c\left(c^{\prime \prime}\right)+1$. Immediately from the definition we have:

Lemma 3.4 Let $c, d \in \mathfrak{C}(G)$. Contributor $d$ can be obtained by activating circles of $c$ if, and only if, $c$ can be obtained by deactivating a circles of $d$. Moreover, the activation/deactivation sets are equal.

Define the activation partial order $\leq_{a}$ where $c \leq_{a} d$ if $d$ is obtained by a sequence of activations starting with $c$, and the activation equivalence relation $\sim_{a}$ where $c \sim_{a} d$ if $c \leq_{a} d$ or $d \leq_{a} c$. The elements of $\mathfrak{C}(G) / \sim_{a}$ are called the activation classes of $G$.

Example 1 Figure 1 is a bidirected graph (with incidences omitted), and some contributors are depicted, sorted by their associated permutation.

Observe that the fifth contributor in (12) is below the (12)(34) contributor in the activation partial order.

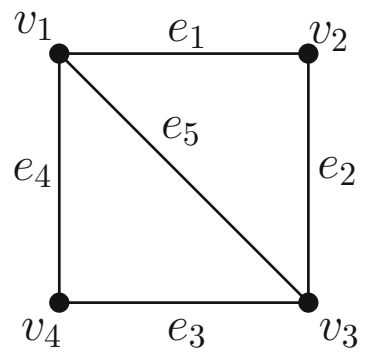

(12)

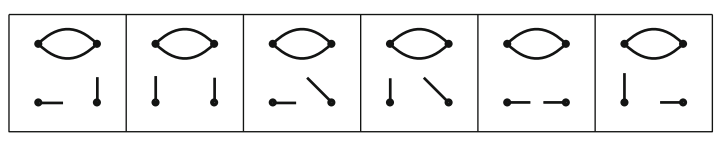

(123)

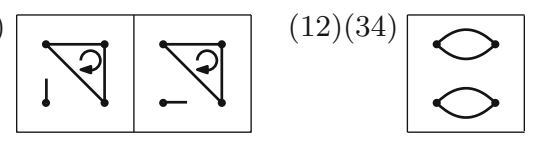

Fig. 1 Understanding contributors 
Lemma 3.5 The minimal elements of activation classes are incomparable, consist of only backsteps, and correspond to the identity permutation.

Lemma 3.6 All activation classes of $G$ are Boolean lattices.

Proof For a given activation class consider the set of possible active circles. The elements of each activation class are ordered by the subsets of active circles, with unique maximal element having all circles active, and unique minimal element having all circle inactive.

We have the following lemma using the facts that (1) every connected component of $G$ is assumed to have an adjacency, and (2) every contributor corresponds to a permutation on the vertices, there is at least one circle that can be activated for each minimal element in each activation class.

Lemma 3.7 Each maximal contributor in a activation class contains at least 1 circle.

Corollary 3.8 Each activation class has at least 2 members.

Example 2 Figure 2 shows 3 activation classes of the graph from Example 1.

The activation classes are ranked by the number of circles, and the minimal element corresponds to the identity permutation.

\subsection{Partitioning activation classes}

For $u, w \in V$ two contributors $c$ and $d$ are $u w$-equivalent, denoted $c \sim_{u w} d$, if $c\left(h_{u}\right)=d\left(h_{u}\right)=w$. Since $\sim_{u w}$ only collects contributors in which the image of $\left(\vec{P}_{1}\right)_{u}$ has head-vertex mapped to $w$ we have:

Lemma $3.9 \mathfrak{C}(G) /\left(\sim_{u_{1} w_{1}} \circ \sim_{u_{2} w_{2}}\right)=\mathfrak{C}(G) /\left(\sim_{u_{2} w_{2}} \circ \sim_{u_{1} w_{1}}\right)$ for $u_{1}, w_{1}, u_{2}, w_{2} \in$ $V$.

As $w$ varies, the composition $\sim_{u \bullet}:=\bigcirc_{w \in V} \sim_{u w}$ is well defined without the need for a total ordering on $V$. Moreover,

Fig. 2 Activation classes are Boolean
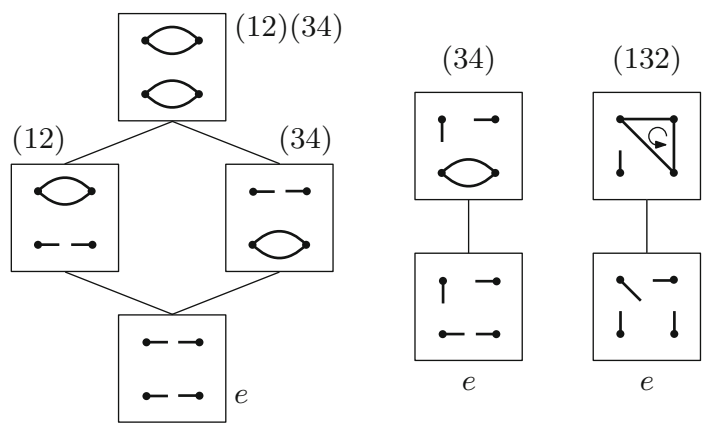
Lemma $3.10 \mathfrak{C}(G) /\left(\sim_{u w} \circ \sim_{a}\right)=\mathfrak{C}(G) /\left(\sim_{a} \circ \sim_{u w}\right)$ or any $u, w \in V$.

By construction, the relation $\sim_{u w} \circ \sim_{a}$ (subsequently, $\sim_{u \bullet} \circ \sim_{a}$ ) refines each $\mathcal{A} \in \mathfrak{C}(G) / \sim_{a}$. Let $\mathcal{A} / \sim_{u w}$ and $\mathcal{A} / \sim_{u \bullet}$ denote the refinement of $\mathcal{A}$ by $\sim_{u w}$ or $\sim_{u \bullet}$, respectively.

Theorem $3.11 \mathfrak{C}(G) /\left(\sim_{u \bullet} \circ \sim_{a}\right)$ is a refinement of each activation class in $\mathfrak{C}(G) / \sim_{a}$ into two principal order ideals (one upper and one lower, with the upper order ideal possibly empty) that are Boolean complements. Moreover, the upper order ideal of activation class $\mathcal{A}$ is empty if, and only if, $c\left(h_{u}\right)=u$ for all $c \in \mathcal{A}$.

Proof Let $\mathcal{A} \in \mathfrak{C}(G) / \sim_{a}$ and observe that every least element of $\mathcal{A}$ is an adjacency free contributor, so the set of possible maximal elements such that $c\left(h_{u}\right)=u$ is nonempty. Using the definition of activation, the facts that $\mathcal{A}$ is Boolean, and that there is at least one element (the $\mathbf{0}$-element) in each activation class with $h_{u} \rightarrow u$, there is exactly one maximal element with $h_{u} \rightarrow u$, let $M(u ; u ; \mathcal{A})$ be this maximal element. Thus, the principal ideal $\downarrow M(u ; u ; \mathcal{A})$ exists and is necessarily Boolean. Moreover, $\downarrow M(u ; u ; \mathcal{A})=\mathcal{A}$ if, and only if, $c\left(h_{u}\right)=u$ for all contributors $c \in \mathcal{A}$, thus $\mathcal{A} / \sim_{u w}$ is empty for all $w \neq u$.

Since $\mathcal{A}$ is Boolean, if there is a contributor $d$ such that $d\left(h_{u}\right)=w \neq u$, then all contributors of $\mathcal{A}$ with $h_{u} \nrightarrow u$ must have $h_{u} \rightarrow w$, since every edge is a 2-edge. Moreover, if there is a contributor of $\mathcal{A}$ with $h_{u} \rightarrow w$, then there is a unique minimal element with $m\left(h_{u}\right)=w \neq u$, let $m(u ; w ; \mathcal{A})$ be this minimal element (if it exists). By construction, $m(u ; w ; \mathcal{A})$ is the contributor of $\mathcal{A}$ with only the circle containing the $u w$-adjacency active, is a rank 1 element in $\mathcal{A}$, and is the Boolean complement of $M(u ; u ; \mathcal{A})$. Thus, $\mathcal{A}=\downarrow M(u ; u ; \mathcal{A}) \cup \uparrow m(u ; w ; \mathcal{A})$.

The $(u ; w)$-cut of activation class $\mathcal{A}$ is the subclass of $\mathcal{A} / \sim_{u w}$ where each element has $c\left(h_{u}\right)=w$-that is, $\downarrow M(u ; u ; \mathcal{A})$ if $u=w$, or $\uparrow m(u ; w ; \mathcal{A})$ if $u \neq w$ and $m(u ; w ; \mathcal{A})$ exists. Let $U, W \subseteq V$ with $|U|=|W|=k$, and $\mathbf{u}=\left(u_{1}, u_{2}, \ldots, u_{k}\right)$, $\mathbf{w}=\left(w_{1}, w_{2}, \ldots, w_{k}\right)$ be linear orderings of $U$ and $W$ according to their placement in the implied linear ordering of $V$ in the underlying incidence matrix. The (u; w)cut of the activation class $\mathcal{A}$ is the corresponding subclass in $\mathcal{A} / \bigcirc_{i \in[k]} \sim_{u_{i} w_{i}}$. Let $\mathcal{A}(\mathbf{u} ; \mathbf{w} ; G)$ denote the $(\mathbf{u} ; \mathbf{w})$-cut of activation class $\mathcal{A}$, and $\widehat{\mathcal{A}}(\mathbf{u} ; \mathbf{w} ; G)$ be the elements of $\mathcal{A}(\mathbf{u} ; \mathbf{w} ; G)$ with the adjacency or backstep from $u_{i}$ to $w_{i}$ is removed for each $i$. Let $\mathfrak{C}(\mathbf{u} ; \mathbf{w} ; G)$ be the set of all elements in all $\mathcal{A}(\mathbf{u} ; \mathbf{w} ; G)$, and $\widehat{\mathfrak{C}}(\mathbf{u} ; \mathbf{w} ; G)$ be the elements of $\mathfrak{C}(\mathbf{u} ; \mathbf{w} ; G)$ with the adjacency or backstep from $u_{i}$ to $w_{i}$ is removed for each $i$.

Example 3 Figure 3 shows $\left(v_{1}, v_{1}\right)$-cuts of the contribution classes from Fig. 2.

Observe that the first two subclasses are non-trivial Boolean lattices, the final subclass is a trivial Boolean lattices, and the second subclass has an empty upper order ideal. 
Fig. $3\left(v_{1}, v_{1}\right)$-cuts of contribution classes

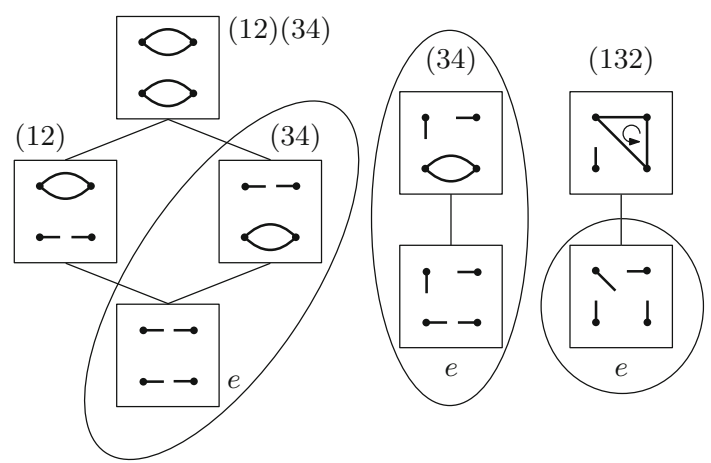

\section{Universal contributors and the matrix-tree theorem}

\subsection{Adjacency completion}

Given an oriented hypergraph $G=(V, E, I, \iota, \sigma)$ let $G^{\prime}=\left(V, E \cup E_{0}, I \cup I_{0}, \iota^{\prime}, \sigma^{\prime}\right)$ be the oriented hypergraph obtained by adding a bidirected edge to $G$ for every nonadjacent pair of vertices, where $\sigma^{\prime}=\sigma$ and $\iota^{\prime}=\iota$ for all $i \in I$, and $\sigma^{\prime}=0$ for all $i \in I_{0}$ (see [15] for relationship to the injective envelope). The sign of a (subcontributor is the product of the weak walks. The inclusion of 0 -signed-incidences in $G^{\prime}$ implies that an element of $\widehat{\mathfrak{C}}(\mathbf{u} ; \mathbf{w} ; G)$ has nonzero sign if, and only if, it exists in $G$. Let $\widehat{\mathfrak{C}}_{\neq 0}\left(\mathbf{u} ; \mathbf{w} ; G^{\prime}\right)$ be the set of nonzero elements of $\widehat{\mathfrak{C}}\left(\mathbf{u} ; \mathbf{w} ; G^{\prime}\right)$. This fact gives the following simple Lemma that relates the global contributors of $G^{\prime}$ to the Chaiken-type forests of [4] separated by multiplicity:

Lemma 4.1 If $U, W \subseteq V$ with linear orderings $\mathbf{u}$ and $\mathbf{w}$, then

$$
\widehat{\mathfrak{C}}_{\neq 0}\left(\mathbf{u} ; \mathbf{w} ; G^{\prime}\right)=\mathfrak{C}(U ; W ; G) .
$$

Example 4 Figure 4 shows an additional contribution class from Fig. 2 that exists in the adjacency completion.

Observe that the (142) contributor with the $v_{4} v_{2}$-adjacency removed is a member of $\widehat{\mathfrak{C}}_{\neq 0}\left(v_{4} ; v_{2} ; G^{\prime}\right)$, exists in $G$, and counts in the $v_{4} v_{2}$-minor calculation.

Lemma 4.1 provides the following restatement of Theorem 2.3:

Theorem 4.2 Let $G$ a bidirected graph with Laplacian matrix $\mathbf{L}_{G}$. Given $U, W \subseteq V$ with $|U|=|W|$ and linear orderings $\mathbf{u}$ and $\mathbf{w}$, let $\left[\mathbf{L}_{G}\right]_{(\mathbf{u} ; \mathbf{w})}$ be the minor of $\mathbf{L}_{G}$ formed by the ordered deletion of the rows corresponding to the vertices in $U$ and the columns corresponding to the vertices in $W$. Then we have,

$$
\begin{aligned}
& \text { 1. } \operatorname{perm}\left(\left[\mathbf{L}_{G}\right]_{(\mathbf{u} ; \mathbf{w})}\right)=\sum_{c \in \widehat{\mathfrak{C}}_{\neq 0}\left(\mathbf{u} ; \mathbf{w} ; G^{\prime}\right)}(-1)^{\text {on }(c)+n n(c),} \\
& \text { 2. } \operatorname{det}\left(\left[\mathbf{L}_{G}\right]_{(\mathbf{u} ; \mathbf{w})}\right)=\sum_{c \in \widehat{\mathfrak{C}}_{\neq 0}\left(\mathbf{u} ; \mathbf{w} ; G^{\prime}\right)} \varepsilon(c) \cdot(-1)^{\text {on }(c)+n n(c) .}
\end{aligned}
$$

where $\varepsilon(c)$ is the number of inversions in the natural bijection from $\bar{U}$ to $\bar{W}$. 

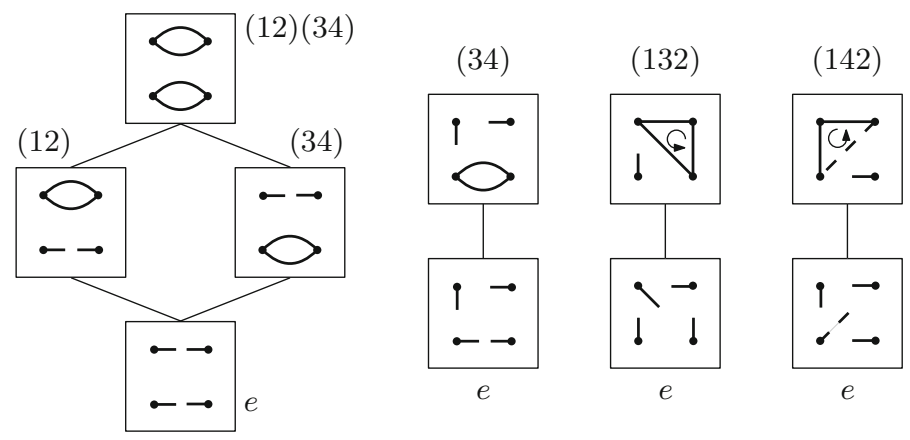

Fig. 4 Contributors in $G^{\prime}$

\subsection{Applications}

We now examine alternate proofs of established results using the Boolean order of contributor maps. Besides providing more insight into contributors, the hope is these techniques can be generalized to a complete theory for oriented hypergraphs - as evidenced by Theorem 2.3.

Let $\mathfrak{M}^{-}$be the set of maximal elements from the positive-circle-free activation classes.

Lemma 4.3 If $G$ is a signed graph, then $\operatorname{det}\left(\mathbf{L}_{G}\right)=\sum_{c \in \mathfrak{M}^{-}} 2^{n c(c)}$.

Proof From Theorem 2.2 and Lemma 3.6

$$
\begin{aligned}
\operatorname{det}\left(\mathbf{L}_{G}\right) & =\sum_{c \in \mathfrak{C}_{\geq 0}(G)}(-1)^{p c(c)} \\
& =\sum_{\mathcal{A} \in \mathfrak{C}^{\prime}(G) / \sim_{a}} \sum_{c \in \mathcal{A}}(-1)^{p c(c)} .
\end{aligned}
$$

Let $M_{\mathcal{A}}^{-}$be the minimal element of activation class $\mathcal{A}$ that has the maximal number of negative circles (if it exists).

Case 1 ( $M_{\mathcal{A}}^{-}$does not exist): If $M_{\mathcal{A}}^{-}$does not exist, then $\mathcal{A}$ is a Boolean lattice with every circle positive. Since each contributor is signed $(-1)^{p c(c)}$, the signs of the contributors of $\mathcal{A}$ alternate with rank. Thus, the sum of elements is the alternating sum of binomial coefficients which equals 0 .

Case 2 ( $M_{\mathcal{A}}^{-}$exists): If $M_{\mathcal{A}}^{-}$exists, it is necessarily unique, $\downarrow M_{\mathcal{A}}^{-}$is Boolean, and every element of $\downarrow M_{\mathcal{A}}^{-}$is signed +1 .

Case $2 a$ (no positive circles): If there are no positive circles in $\mathcal{A}$, then $\downarrow M_{\mathcal{A}}^{-}=\mathcal{A}$ and the sum of the elements is $2^{n c\left(M_{\mathcal{A}}\right)}$. 
Case $2 b$ (at least one positive circle): Suppose $1_{\mathcal{A}}$ has exactly $n$ positive circles, let $p_{1}, p_{2}, \ldots, p_{n}$ be contributors of $\mathcal{A}$ with exactly one positive circle, and let $P_{i}=$ $\left[0_{\mathcal{A}}, p_{i}\right]$. For $k \in[0, n]$, consider the collection of Boolean lattices

$$
B_{k}=\left(\downarrow M_{\mathcal{A}}\right) \vee \bigvee_{i=1}^{k} P_{i},
$$

where $B_{0}=\downarrow M_{\mathcal{A}}$. By construction, $B_{i} \cong B_{i} \vee p_{i+1}, B_{i+1}=B_{i} \vee P_{i+1}, B_{i+1}$ is a Boolean lattice 1 rank larger than $B_{i}$, and $B_{n}=\mathcal{A}$. Additionally, for each $b \in B_{i}$ and $b \vee p_{i+1} \in B_{i} \vee p_{i+1}$,

$$
(-1)^{p c\left(b \vee p_{i+1}\right)}=(-1)^{p c(b)+1}
$$

Thus, the sum of all contributors is necessarily 0 as long as there exists a positive circle.

The only non-cancellative activation classes are those that are positive-circle-free, and the result follows with the observation that an empty sum is 0 .

Corollary 4.4 If $G$ is a balanced signed graph, then $\operatorname{det}\left(\mathbf{L}_{G}\right)=0$.

Let $\widehat{\mathcal{A}}_{\neq 0}\left(u ; w ; G^{\prime}\right)$ be the set of nonzero elements of $\widehat{\mathcal{A}}\left(u ; w ; G^{\prime}\right)$.

Lemma 4.5 If $G$ is a bidirected graph, then the set of elements in all single-element $\widehat{\mathcal{A}}_{\neq 0}\left(u ; w ; G^{\prime}\right)$ is activation equivalent to the set of spanning trees of $G$.

Proof Case $1 a(u=w)$ : Suppose $u=w$, and let $\widehat{\mathcal{A}}_{\neq 0}\left(u ; u ; G^{\prime}\right)$ be a single-element activation classes of $G^{\prime}$. The element of $\widehat{\mathcal{A}}_{\neq 0}\left(u ; u ; G^{\prime}\right)$ consists of exactly $|V|-1$ backsteps, all of which exist in $G$, but none of which contain $u$. Unpacking all backsteps results in a circle-free subgraph on $|V|$ vertices with $|V|-1$ edges, i.e., a spanning tree-if it was not circle-free then $\widehat{\mathcal{A}}_{\neq 0}\left(u ; u ; G^{\prime}\right)$ would have more than a single element. Thus, the cardinality of the set of single-element $\widehat{\mathcal{A}}_{\neq 0}\left(u ; u ; G^{\prime}\right)$ is less than, or equal to, the number spanning trees of $G$.

Case $1 b(u=w)$ : Now consider all spanning outward arborescences of $G$ rooted at $u$. For each arborescence, pack all adjacencies along the opposite orientation of the arborescence to produce a unique, nonzero, element of an $\widehat{\mathcal{A}}\left(u ; u ; G^{\prime}\right)$. Thus, the cardinality of the set of single-element $\widehat{\mathcal{A}}_{\neq 0}\left(u ; u ; G^{\prime}\right)$ is greater than, or equal to, the number spanning trees of $G$.

Case $2 a(u \neq w)$ : Suppose $u \neq w$, and let $\widehat{\mathcal{A}}_{\neq 0}\left(u ; w ; G^{\prime}\right)$ be a single-element activation class of $G^{\prime}$. Since $\widehat{\mathcal{A}}_{\neq 0}\left(u ; w ; G^{\prime}\right)$ is obtained by the upper order ideal of $\mathcal{A}\left(u ; w ; G^{\prime}\right)$ generated by the maximal contributor $M(u ; w ; \mathcal{A})$, and $\widehat{\mathcal{A}}_{\neq 0}\left(u ; w ; G^{\prime}\right)$ consists of a single element, $M(u ; w ; \mathcal{A})$ must be unicyclic. Thus, the corresponding contributor in $\widehat{\mathcal{A}}_{\neq 0}\left(u ; w ; G^{\prime}\right)$ must contain a $w u$-path on $k$ vertices, and backsteps at the $|V|-k$ vertices outside the path. Unpacking all backsteps is a circle-free subgraph on $|V|$ vertices and $|V|-1$ edges - if not then $\widehat{\mathcal{A}}_{\neq 0}\left(u ; w ; G^{\prime}\right)$ would have more than a single element. Thus, the cardinality of the set of single-element $\widehat{\mathcal{A}}_{\neq 0}\left(u ; w ; G^{\prime}\right)$ is less than, or equal to, the number spanning trees of $G$. 
Case $2 b(u \neq w)$ : Now consider the set of spanning trees of $G$. To see that the cardinality of the set of single-element $\widehat{\mathcal{A}}_{\neq 0}\left(u ; w ; G^{\prime}\right)$ is greater than, or equal to, the number spanning trees of $G$, examine the following sub-cases:

Case $2 b$, part $1(u \neq w)$ : If a spanning tree $T$ contains an adjacency between $u$ and $w$ construct the unicyclic-contributor of $G$ as follows: (1) add another parallel adjacency between $u$ and $w,(2)$ orient the parallel edges to form a degenerate 2-circle, and (3) pack all remaining adjacencies away from $u$ and $w$. The member of $\widehat{\mathcal{A}}_{\neq 0}\left(u ; w ; G^{\prime}\right)$ is obtained by deleting the $u w$-directed adjacency.

Case $2 b$, part $2(u \neq w)$ : If a spanning tree $T$ does not contain an adjacency between $u$ and $w$ construct the unicyclic-contributor of $G$ as follows: (1) add a $u w$-directed adjacency, (2) oriented the resulting unique fundamental circle coherently with the $u w$-directed adjacency, and (3) pack all remaining adjacencies away from the fundamental circle. The member of $\widehat{\mathcal{A}}_{\neq 0}\left(u ; w ; G^{\prime}\right)$ is obtained by deleting the $u w$-directed adjacency.

Using the techniques of the previous two Lemmas, adjusting for the cofactor, and using the fact that every adjacency (hence, circle) is positive in a graph, it is easy to reclaim:

Corollary 4.6 If $G$ is a graph, then the uw-cofactor of $\mathbf{L}_{G}$ is $T(G)$, the number of spanning trees of $G$.

Acknowledgements The authors sincerely thank the referee for their careful reading of the manuscript and for their valuable feedback.

\section{References}

1. Belardo, F., Simić, S.: On the Laplacian coefficients of signed graphs. Linear Algebra Appl. 475, 94-113 (2015). https://doi.org/10.1016/j.laa.2015.02.007

2. Berge, C.: Hypergraphs, North-Holland Mathematical Library, vol. 45. North-Holland Publishing Co., Amsterdam (1989). Combinatorics of finite sets (Transl: French)

3. Bronski, J.C., DeVille, L.: Spectral theory for dynamics on graphs containing attractive and repulsive interactions. SIAM J. Appl. Math. 74(1), 83-105 (2014)

4. Chaiken, S.: A combinatorial proof of the all minors matrix tree theorem. SIAM J. Algebr. Discrete Methods 3(3), 319-329 (1982)

5. Chaiken, S., Kleitman, D.: Matrix tree theorems. J. Comb. Theory Ser. A 24(3), 377-381 (1978)

6. Chen, G., Liu, V., Robinson, E., Rusnak, L.J., Wang, K.: A characterization of oriented hypergraphic Laplacian and adjacency matrix coefficients. arXiv:1704.03599 [math.CO] (2017)

7. Chen, V., Rao, A., Rusnak, L., Yang, A.: A characterization of oriented hypergraphic balance via signed weak walks. Linear Algebra Appl. 485, 442-453 (2015)

8. Chung, F.R.K., Langlands, R.P.: A combinatorial Laplacian with vertex weights. J. Comb. Theory Ser. A 75(2), 316-327 (1996)

9. Conforti, M., Cornuéjols, G., Rao, M.R.: Decomposition of balanced matrices. J. Comb. Theory Ser. B 77(2), 292406 (1999)

10. Conforti, M., Cornuéjols, G., Vušković, K.: Balanced matrices. Discrete Math. 306(19-20), 2411-2437 (2006). https://doi.org/10.1016/j.disc.2005.12.033

11. Cvetkovic, D., Doob, M., Sachs, H.: Spectra of Graphs: Theory and Applications, 3rd edn. Oxford Science Publications, Oxford (1998)

12. Edmonds, J., Johnson, E.L.: Matching: a well-solved class of integer linear programs. In: Combinatorial Structures and Their Applications (Proc. Calgary Internat., Calgary, Alta., 1969), pp. 89-92. Gordon and Breach, New York (1970) 
13. Fulkerson, D., Hoffman, A., Oppenheim, R.: On balanced matrices. Math. Program. Study 1, 120-132 (1974)

14. Graovac, A., Gutman, I., Trinajstić, N., Živković, T.: Graph theory and molecular orbitals. Theoret. Chimica Acta 26(1), 67-78 (1972)

15. Grilliette, W., Seacrest, D., Seacrest, T.: On blow-ups and injectivity of quivers. Electron. J. Comb. 20(2), 40 (2013)

16. Levine, L.: Sandpile groups and spanning trees of directed line graphs. J. Comb. Theory Ser. A 118(2), 350-364 (2011)

17. Reff, N.: Spectral properties of complex unit gain graphs. Linear Algebra Appl. 436(9), 3165-3176 (2012). https://doi.org/10.1016/j.laa.2011.10.021

18. Reff, N., Rusnak, L.: An oriented hypergraphic approach to algebraic graph theory. Linear Algebra Appl. 437(9), 2262-2270 (2012). https://doi.org/10.1016/j.laa.2012.06.011

19. Rusnak, L.: Oriented hypergraphs: introduction and balance. Electron. J. Comb. 20 (3), 48 (2013)

20. Schrijver, A.: Combinatorial Optimization: Polyhedra and Efficiency, Vol. A-C, Algorithms and Combinatorics, vol. 24. Springer, Berlin (2004)

21. Shi, C.J.: A signed hypergraph model of the constrained via minimization problem. Microelectron. J. 23(7), 533-542 (1992). https://doi.org/10.1016/0026-2692(92)90064-8

22. Shi, C.J., Brzozowski, J.A.: A characterization of signed hypergraphs and its applications to VLSI via minimization and logic synthesis. Discrete Appl. Math. 90(1-3), 223-243 (1999). https://doi.org/10. 1016/S0166-218X(98)00092-4

23. Tutte, W.T.: Graph Theory, Encyclopedia of Mathematics and Its Applications, vol. 21. AddisonWesley Publishing Company Advanced Book Program, Reading, MA, With a foreword by C. St. J. A, Nash-Williams (1984)

24. Wang, Y., Gope, D., Jandhyala, V., Shi, C.J.: Generalized Kirchoff's current and voltage law formulation for coupled circuit-electromagnetic simulation with surface integral equations. IEEE Trans. Microwave Theory Tech. 52(7), 1673-1682 (2004)

25. Zaslavsky, T.: Signed graphs. Discrete Appl. Math. 4(1), 47-74 (1982). https://doi.org/10.1016/0166218X(82)90033-6. (Erratum, ibid., 5 (1983), 248)

26. Zaslavsky, T.: Orientation of signed graphs. Eur. J. Combin. 12(4), 361-375 (1991) 